\title{
Fosfolipasa $\mathrm{A}_{2}$ asociada a lipoproteínas y patología vascular cerebral
}

\author{
F.J. Álvarez-Pérez ${ }^{\text {a,b }}$, I. Verde ${ }^{a}$
}

FOSFOLIPASA $A_{2}$ ASOCIADA A LIPOPROTEÍNAS Y PATOLOGÍA VASCULAR CEREBRAL

\begin{abstract}
Resumen. Introducción. La fosfolipasa $A_{2}$ asociada a lipoproteínas ( $\left.F L A_{2}-L p\right)$ es una enzima perteneciente a la superfamilia de las fosfolipasas $A_{2}$. El $80 \%$ circula unido al colesterol ligado a lipoproteínas de baja densidad (colesterol-LDL), con cuyos niveles plasmáticos se correlaciona positivamente. Su función principal es hidrolizar los fosfolípidos oxidados del colesterol$L D L$, formando derivados proinflamatorios en la placa del ateroma. Múltiples estudios han relacionado el aumento de $F L A_{2}-L p$ y el riesgo vascular. Objetivo. Revisar los datos disponibles sobre la relación entre $F L A_{2}-L p$ y la patología vascular, específicamente el ictus. Desarrollo. Diferentes estudios de cohortes y de casos y controles han demostrado una asociación entre los niveles superiores de $\mathrm{FLA}_{2}$-Lp y el riesgo de sufrir un primer evento cardiovascular (cardiopatía isquémica, ictus isquémico) o su recurrencia, la progresión de la lesión coronaria evaluada mediante tomografía computarizada cardíaca y la muerte de causa cardiovascular. Las medidas de riesgo publicadas son inferiores a 2, y se ha demostrado que los niveles elevados de la enzima son un factor de riesgo independiente de los factores de riesgo cardiovascular clásicos y de los niveles de colesterol no ligado a lipoproteínas de alta densidad y de proteína $C$ reactiva. Los datos clínicos y el conocimiento de su papel en la desestabilización de la placa han justificado el desarrollo de fármacos inhibidores. Conclusiones. La evidencia existente permite considerar a los pacientes en el rango superior de $F L A_{2}-L p$ como sujetos con mayor riesgo de padecer un episodio vascular. La aplicación al ictus isquémico precisa definir mejor su papel fisiopatológico y conocer los resultados de ensayos clínicos de fase II actualmente en curso en cardiopatía isquémica. [REV NEUROL 2009; 49: 88-94]
\end{abstract}

Palabras clave. Ateroesclerosis. Cardiopatía isquémica. Colesterol. Fosfolipasa $A_{2}$. Ictus. Proteína C reactiva.

\section{INTRODUCCIÓN}

La estrategia fundamental en el tratamiento de la patología ateroesclerótica implica el control agresivo de los factores de riesgo clásicos modificables, como la diabetes, la hipertensión arterial, el consumo de tabaco o la obesidad, entre otros. Sin embargo, la efectividad limitada de estas medidas ha condicionado la búsqueda de nuevas vías en la fisiopatología de la ateroesclerosis que permitan el desarrollo posterior de nuevos tratamientos. Se ha establecido que la inflamación es uno de los mecanismos asociado a todos los estadios de la ateroesclerosis, al estar implicada tanto en sus fases iniciales como durante su progresión debida a la desestabilización de la placa. Actualmente se dispone de marcadores de inflamación determinables de una forma relativamente sencilla en la práctica clínica habitual, como la proteína $\mathrm{C}$ reactiva (PCR) o el fibrinógeno. Sin embargo, no se realiza un tratamiento específico del estado inflamatorio subyacente en los sujetos de riesgo, con la excepción de posibles efectos colaterales antiinflamatorios de fármacos como las estatinas [1].

Uno de los marcadores de inflamación que se han asociado recientemente al aumento del riesgo cardiovascular es la fosfolipasa $\mathrm{A}_{2}$ asociada a lipoproteínas $\left(\mathrm{FLA}_{2}-\mathrm{Lp}\right)$. Diferentes estudios en los que se ha determinado tanto su masa total como su actividad enzimática han demostrado su relación directa con el riesgo de muerte, de eventos coronarios y de ictus. Esta asociación no sólo se ha observado al realizar el análisis univariado, sino que se ha mantenido después de aplicar el multivariado, con medidas de riesgo próximas a 2 . En algunos estudios se ha

\footnotetext{
Aceptado tras revisión externa: 23.02.09.

${ }^{a}$ Departamento de Medicina. Facultad de Ciencias de la Salud. Universidad de Beira Interior. ${ }^{b}$ Unidad de Ictus. Hospital de Covilhã. Covilhã, Portugal.

Correspondencia: Dr. Francisco José Álvarez Pérez. Departamento de Medicina. Facultad de Ciencias de la Salud. Universidad de Beira Interior. Avda. Infante D. Henrique, 6200-506. Covilhã, Portugal. Fax: 351-275-329-099. E-mail: franciscoplus@hotmail.com

(C) 2009, REVISTA DE NEUROLOGÍA
}

demostrado una relación positiva entre la $\mathrm{FLA}_{2}$-Lp y el colesterol ligado a lipoproteínas de baja densidad (colesterol-LDL), justificada por el hecho de que este último es el principal transportador del enzima.

El número de artículos originales y revisiones publicados durante los últimos años sobre el riesgo vascular asociado a la $\mathrm{FLA}_{2}$-Lp es creciente [2-5]. Los trabajos se refieren mayoritariamente al riesgo de patología coronaria, y proporcionalmente son menores los referentes al ictus. Como es habitual en la investigación médica, existen controversias sobre el valor real de la $\mathrm{FLA}_{2}-\mathrm{Lp}$, ya que existen estudios que han demostrado una falta de asociación entre sus niveles circulantes y la incidencia de patología cardiovascular. Dada la potencial importancia de este nuevo marcador, el objetivo del presente trabajo es revisar tanto las características fundamentales de la $\mathrm{FLA}_{2}$-Lp como la información disponible acerca de su relación con la patología vascular, y específicamente con la cerebrovascular.

\section{CARACTERÍSTICAS DE LA FLA 2 -Lp}

La FLA 2 -Lp, también conocida como acetilhidrolasa del factor activador de plaquetas (FAP), pertenece a la gran superfamilia de enzimas fosfolipasa $\mathrm{A}_{2}$. Estas enzimas se caracterizan por su capacidad para catalizar específicamente la hidrólisis de la cadena éster central (sn-2) de los fosfolípidos, entre los que se encuentra el FAP, implicado en la lesión secundaria en diferentes modelos de isquemia [6,7]. La hidrólisis genera como productos finales ácidos grasos libres y lisofosfolípidos. Además de su función como reserva energética, algunos ácidos grasos libres pueden actuar como segundos mensajeros y otros como mediadores de la inflamación. Los lisofosfolípidos intervienen básicamente en la señalización celular, en el remodelamiento de los fosfolípidos y en el daño de membrana.

Los primeros estudios sobre la actividad enzimática de las $\mathrm{FLA}_{2}$ se realizaron a finales del siglo XIX utilizando veneno de serpiente, y la investigación posterior permitió determinar tam- 
bién su presencia en fluidos biológicos de otras especies, incluyendo la humana. Estas enzimas son dependientes del calcio y, mientras que las formas secretadas son ricas en puentes disulfuro y poseen residuos catalíticos de histidina y aspartato, las formas citosólicas descritas en neutrófilos y plaquetas no poseen estos puentes y su residuo catalítico está constituido por serina. Diferentes criterios permiten clasificarlas en 11 grupos, para lo que se remite al lector a revisiones especializadas [8].

La $\mathrm{FLA}_{2}$-Lp circulante se produce fundamentalmente en el tejido hematopoyético. Aproximadamente, el $80 \%$ de la enzima circula unida a la apolipoproteína B del colesterol-LDL y el $20 \%$ restante lo hace unido al colesterol ligado a lipoproteínas de alta densidad (colesterol-HDL). Otros tejidos pueden también sintetizar $\mathrm{FLA}_{2}$-Lp, específicamente los macrófagos y los linfocitos $T$ que se pueden localizar en la placa de ateroma. Se ha considerado que su función biológica podía ser tanto anti como proaterogénica. Su papel antiaterogénico se basaría en la degradación de fosfolípidos oxidados en la molécula de LDL (eliminando así el componente de LDL que se ha demostrado asociado a la vulnerabilidad de placa, a la disfunción endotelial y a la quimiotaxis y adhesión de los monocitos al endotelio) y en la degradación del FAP. Esta función no se ha establecido firmemente, ya que la degradación del FAP no se ha demostrado in vivo, y se ha descrito que ciertos fosfolípidos oxidados pueden ejercer un papel antiinflamatorio $[9,10]$. Actualmente, se considera que su papel proaterogénico está definido de una forma más sólida. En teoría, comienza por la formación de mediadores inflamatorios a partir de la degradación enzimática de los fosfolípidos oxidados contenidos en el colesterol-LDL. Esta hidrólisis forma lisofosfatidilcolina y ácidos grasos oxidados no esterificados. El primero de ellos promueve la apoptosis y la quimiotaxis de monocitos, linfocitos $\mathrm{T}$ y neutrófilos en el endotelio y el músculo liso. A su vez, los ácidos grasos oxidados no esterificados participan en la quimiotaxis de monocitos. Diferentes hallazgos, como la inhibición de la quimiotaxis monocitaria mediante el bloqueo selectivo de $\mathrm{FLA}_{2}$-Lp y la existencia del receptor $\mathrm{G}_{2} \mathrm{~A}$ para lisofosfatidilcolina en macrófagos y linfocitos presentes en lesiones ateroescleróticas humanas, apoyan este papel proaterogénico.

Se ha valorado el riesgo vascular asociado a $\mathrm{FLA}_{2}-\mathrm{Lp}$ en función del análisis de sus polimorfismos en diferentes poblaciones, con resultados contradictorios. Por ejemplo, en japoneses portadores de la variante Val279Phe [11], que reduce al menos un $27 \%$ el nivel sérico de la enzima, se ha descrito un aumento del riesgo vascular, frente a una reducción del riesgo de infarto agudo de miocardio (IAM) en sujetos caucasianos portadores de la variante Ala379Val, que causa una disminución de la afinidad de $\mathrm{FLA}_{2}$-Lp por el FAP exógeno [12].

Se ha descrito que los niveles de $\mathrm{FLA}_{2}$-Lp son superiores en hombres y se correlacionan positivamente con la presión arterial sistólica, el índice de masa corporal, el colesterol total y no HDL y con el recuento de leucocitos, mientras que la correlación es negativa en el caso del colesterol-HDL y el consumo de alcohol $[13,14]$. Los niveles parecen ser independientes de la edad, presión arterial diastólica, diabetes, consumo de tabaco y niveles de PCR. Otros estudios, sin embargo, no hallaron correlación entre la $\mathrm{FLA}_{2}$-Lp y el índice de masa corporal, consumo de tabaco, diabetes, hipertensión arterial y edad [15-17]. La correlación positiva con los niveles de colesterol total y no HDL (en la práctica ,equivalente al LDL) es la asociación más sólida, descrita en prácticamente todos los estudios, y se justifica por la fisiología de la enzima.
Es posible determinar tanto la masa de $\mathrm{FLA}_{2}$-Lp, mediante enzimoinmunoensayo (PLAC ${ }^{\circledR}$ test, Dia Dexus Inc), que permite una determinación cuantitativa, como la actividad enzimática [3].

\section{ESTUDIOS DE LA RELACIÓN ENTRE FLA 2 -Lp Y PATOLOGÍA CARDIOVASCULAR, SIN INCLUIR EL ICTUS}

La mayor parte de los datos acerca del papel proaterogénico de la $\mathrm{FLA}_{2}$-Lp proceden de pacientes con cardiopatía isquémica. Uno de los estudios más relevantes utilizó la cohorte del West of Scotland Coronary Prevention Study [15]. En él se reclutó a 6.595 varones entre febrero de 1989 y mayo de 1995. Los sujetos debían presentar niveles de colesterol-LDL de 174-232 mg/dL y no referir una historia previa de cardiopatía isquémica. Se aleatorizaron para recibir $40 \mathrm{mg} /$ día de pravastatina o placebo. El criterio de valoración principal fue la incidencia combinada de IAM y muerte de origen coronario. Se observó que la terapia con pravastatina se asociaba a una disminución del $31 \%$ en este objetivo, y la reducción de procedimientos de revascularización coronaria era similar. Los autores compararon los niveles de PCR de alta sensibilidad (PCR-as) y de FLA 2 -Lp de 580 pacientes que habían experimentado un IAM (503) o que se habían sometido a un proceso de revascularización $(n=77)$ con los de 1.160 controles de edad similar. Como en otros estudios, se observó una correlación positiva entre los niveles de $\mathrm{FLA}_{2}$-Lp y de colesterol-LDL y, en menor grado e igualmente positiva, con los niveles de fibrinógeno. Se demostró que la enzima era prácticamente independiente de otros factores de riesgo, incluyendo la PCR-as y el consumo de tabaco. En el análisis univariado, los niveles elevados $\mathrm{FLA}_{2}$-Lp, PCR-as y fibrinógeno, y el recuento leucocitario aumentado, se asociaron a un mayor riesgo del objetivo combinado de IAM/muerte súbita o necesidad de revascularización. El riesgo se mantuvo para la $\mathrm{FLA}_{2}$-Lp después de realizar un análisis ajustado en función de los factores de riesgo tradicionales y de los marcadores inflamatorios. Se determinó una razón de riesgos (RR) de 1,18, con un intervalo de confianza del 95\% (IC 95\%) de 1,05-1,33 para cada aumento de 1 desviación estándar. Este riesgo fue claramente superior entre los sujetos tratados con pravastatina $(\mathrm{RR}=1,67$; IC 95\% $=1,24$ $2,25)$ que entre los no tratados ( $\mathrm{RR}=1,17$; IC 95\% = 0,9-1,51). En la discusión, los autores destacan su papel independiente como factor de riesgo coronario, complementario al de los marcadores de riesgo ya conocidos, y sugieren su utilización complementaria en la estratificación del riesgo y su posible inhibición directa como objetivo terapéutico.

En otro estudio se describió un mayor riesgo de muerte durante el primer año posterior al IAM en los pacientes cuyos niveles de $\mathrm{FLA}_{2}$-Lp se situaban en el tercil medio y superior respecto al inferior, con $\mathrm{RR}$ de 2,2 (IC 95\% = 0,88-5,54) y 4,93 (IC 95\% = 2,1-11,6), respectivamente [18].

Numerosos trabajos adicionales confirmaron la asociación entre $\mathrm{FLA}_{2}$-Lp y cardiopatía isquémica [19-22], e incluso se ha relacionado con la progresión de la vasculopatía asociada al transplante cardíaco [23] y con la presencia de patología coronaria en coronariografía, aunque esta última asociación se perdió al realizar el análisis ajustado por otros factores de riesgo cardiovascular [16].

Aunque la mayor parte de los estudios publicados apoyan el papel de $\mathrm{FLA}_{2}$-Lp como marcador de riesgo cardiovascular, existen trabajos con resultados contradictorios. Un estudio re- 
Tabla I. Estudios que evalúan la relación entre $\mathrm{FLA}_{2}-\mathrm{Lp}$ y el riesgo de patología coronaria, sin incluir al ictus.

\begin{tabular}{|c|c|c|c|c|c|c|}
\hline Ref. & Diseño del estudio & ECV valorados & $\begin{array}{l}\text { Características } \\
\text { de los individuos }\end{array}$ & $\begin{array}{l}\mathrm{N} \cdot{ }^{\circ} \text { de } \\
\text { individuos }\end{array}$ & $\begin{array}{l}\text { Seguimiento } \\
\text { medio (años) }\end{array}$ & Riesgo \\
\hline [15] & Casos y controles & Eventos coronarios & $\begin{array}{l}\text { Sujetos con hiper- } \\
\text { colesterolemia }\end{array}$ & 1.740 & 4,9 & $\begin{array}{l}\mathrm{RR}=1,18(\mathrm{IC} 95 \%=1,05-1,33) \\
\text { por cada aumento de } 1 \mathrm{DE}\end{array}$ \\
\hline [42] & Casos y controles & Eventos coronarios & Pacientes con $\mathrm{Cl}$ & 973 & & $\begin{array}{l}\mathrm{OR}=1,8(\mathrm{IC} 95 \%=1,01-3,2) \\
\text { al comparar Q4 con Q1 }\end{array}$ \\
\hline [35] & Cohorte prospectiva & Eventos coronarios & Sujetos sanos & 934 & 14 & $\begin{array}{l}\mathrm{OR}=1,23(\mathrm{IC} 95 \%=1,02-1,47) \\
\text { por cada aumento de } 1 \mathrm{DE}\end{array}$ \\
\hline [38] & Casos y controles & $\begin{array}{l}\text { Placas coronarias } \\
\text { calcificadas }\end{array}$ & $\begin{array}{l}\text { Sujetos de } \\
18-30 \text { años }\end{array}$ & 532 & 15 & $\begin{array}{l}\mathrm{OR}=1,28(\mathrm{IC} 95 \%=1,03-1,6) \\
\text { por cada aumento de } 1 \text { DE }\end{array}$ \\
\hline [33] & Casos y controles & $\begin{array}{l}\text { Lesión en } \\
\text { coronariografía }\end{array}$ & Pacientes con $\mathrm{Cl}$ & 3.148 & & $\mathrm{OR}=1,31(\mathrm{IC} 95 \%=1,19-1,43)$ por $\mathrm{Q}$ \\
\hline [19] & Casos y controles & $\begin{array}{l}\text { Lesión en } \\
\text { coronariografía }\end{array}$ & Pacientes con $\mathrm{Cl}$ & 791 & & $\begin{array}{l}\mathrm{OR}=1,91(\mathrm{IC} 95 \%=1,12-3,28) \\
\text { al comparar } \mathrm{Q} 4 \text { con } \mathrm{Q} 1\end{array}$ \\
\hline [36] & Cohorte prospectiva & Eventos coronarios & Pacientes con $\mathrm{Cl}$ & 766 & 2,2 & $\begin{array}{l}\mathrm{RR}=1,9(\mathrm{IC} 95 \%=1,31-2,75) \\
\text { al comparar Q4 con } \mathrm{Q} 1\end{array}$ \\
\hline [34] & Cohorte prospectiva & $\begin{array}{l}\text { Mortalidad y } \\
\text { progresión en } \\
\text { coronariografía }\end{array}$ & Pacientes con $\mathrm{Cl}$ & 1.493 & 6,7 & $\begin{array}{l}\mathrm{RR}=1,15(\mathrm{IC} 95 \%=0,78-1,71) \text { para mayor lesión } \\
\text { en coronariografía y } \mathrm{RR}=1,27 \text { (IC } 95 \%=0,58-2,78) \\
\text { para mortalidad cardiovascular al comparar Q1 y } \\
\text { Q2; RR superiores al comparar Q1 con Q3 y Q4 }\end{array}$ \\
\hline [18] & Casos y controles & Mortalidad & Pacientes con $\mathrm{Cl}$ & 271 & 1 & $\begin{array}{l}\mathrm{RR}=2,2(\mathrm{IC} 95 \%=0,88-5,54) \text { y } 4,93(\mathrm{IC} 95 \%= \\
2,1-11,6) \text { al comparar T1 con T2 y T3 de } \mathrm{FLA} \mathrm{A}_{2}-\mathrm{Lp}, \\
\text { respectivamente }\end{array}$ \\
\hline [17] & Cohortes & $\begin{array}{l}\text { IAM y mortalidad } \\
\text { a } 30 \text { días y } 2 \text { años }\end{array}$ & Pacientes con $\mathrm{Cl}$ & 2.701 & 2 & $\begin{array}{l}\text { Ausencia de asociación entre } F L A_{2}-L p \text { y el riesgo } \\
\text { de } \mathrm{Cl} \text { y la gravedad de la enfermedad coronaria }\end{array}$ \\
\hline [21] & Cohorte prospectiva & Eventos coronarios & $\begin{array}{l}\text { Pacientes ingre- } \\
\text { sados por SCA }\end{array}$ & 429 & $\begin{array}{l}42 \text { días } \\
\text { post-SCA }\end{array}$ & $\begin{array}{l}\mathrm{RR}=2,6(\mathrm{IC} 95 \%=1,1-6,6) \text { para pacientes con } \\
\mathrm{FLA}_{2}-\mathrm{Lp}>210 \mu \mathrm{g} / \mathrm{L} \text { y BNP moderadamente alto }\end{array}$ \\
\hline [41] & Casos y controles & $\begin{array}{l}\text { Mortalidad de causa } \\
\text { coronaria }\end{array}$ & $\begin{array}{l}\text { Pacientes con } \\
\text { lesión coronaria }\end{array}$ & 3.232 & 5,5 & $\begin{array}{l}\mathrm{RR}=2(\mathrm{IC} 95 \%=1,4-3,1) \text { para pacientes en T3 } \\
\text { respecto a T1; estratificado según PCR }\end{array}$ \\
\hline$[20]$ & Cohorte prospectiva & Eventos coronarios & $\begin{array}{l}\text { Ancianos aparen- } \\
\text { temente sanos }\end{array}$ & 1.077 & 16 & $\begin{array}{l}\mathrm{RR}=1,66,1,8 \text { y } 1,89 \text { al comparar } \mathrm{Q} 4 \text { con } \mathrm{Q} 1, \\
\mathrm{Q} 2 \text { y } \mathrm{Q} 3 \text {, respectivamente }\end{array}$ \\
\hline [23] & Cohorte prospectiva & Eventos coronarios & $\begin{array}{l}\text { Pacientes sometidos } \\
\text { a trasplante cardíaco }\end{array}$ & 112 & 5,1 & $\begin{array}{l}\mathrm{RR}=2,4(\mathrm{IC} 95 \%=1,16-5,19) \\
\text { si } F L A_{2}-L p>236 \mathrm{ng} / \mathrm{mL}\end{array}$ \\
\hline
\end{tabular}

BNP: péptido natriurético tipo B; Cl: cardiopatía isquémica; DE: desviación estándar; ECV: eventos cardiovasculares; FLA -Lp: fosfolipasa $A_{2}$ asociada a lipoproteínas; IAM: infarto agudo de miocardio; IC: intervalo de confianza; OR: odds ratio; PCR: proteína C reactiva; Q (Q1-Q4): cuartil (cuartiles 1-4); RR: razón de riesgos; SCA: síndrome coronario agudo; T (T1-T3): tercil (terciles 1-3).

ciente demostró que, si bien los pacientes con síndromes coronarios agudos presentaban niveles superiores de $\mathrm{FLA}_{2}$-Lp que los controles y que estos niveles se correlacionaban con los de lípidos, no existía relación entre los niveles de la enzima y otros marcadores establecidos de riesgo cardiovascular, el riesgo de mortalidad y el desarrollo de nuevos eventos coronarios [17].

La tabla I resume los principales estudios publicados sobre $\mathrm{FLA}_{2}$-Lp y patología coronaria.

\section{ESTUDIOS DE LA RELACIÓN ENTRE FLA 2 -LP E ICTUS ISQUÉMICO}

Diferentes trabajos publicados en los años noventa describieron niveles plasmáticos significativamente superiores de la hidrolasa del FAP en pacientes con ictus isquémico comparados con controles. Los autores consideraron que este hecho podía relacionarse con la adaptación del organismo a la mayor síntesis de FAP en el tejido cerebral sometido a isquemia [24,25].

Durante los años siguientes, diferentes estudios de cohortes permitieron asociar los niveles superiores de $\mathrm{FLA}_{2}$-Lp con el aumento del riesgo de sufrir un ictus isquémico. Aunque la mayor parte de ellos se realizaron en pacientes con cardiopatía isquémica y la incidencia de ictus era uno de los criterios de valoración secundarios, algunos se diseñaron específicamente para estudiar el ictus isquémico. Uno de los trabajos más relevantes es el Atherosclerosis Risk in Communities, que incluyó a 15.792 adultos de 45 a 64 años [14]. En él se realizó un primer examen basal entre 1987-1989, y tres exámenes posteriores hasta 1998. Después de aplicar los diferentes criterios de exclusión, el número total de sujetos disponibles para el análisis fue de 12.762. Se determinaron los niveles de $\mathrm{FLA}_{2}$-Lp masa y PCR-as en los individuos que experimentaron un ictus isquémico durante el seguimiento y en una cohorte aleatoria integrada por otros 784 participantes.

Los investigadores detectaron 223 ictus, de los que 194 eran isquémicos. Tanto la prevalencia de factores de riesgo cardiovascular tradicionales, como hipertensión, diabetes y consumo de tabaco, como los niveles de triglicéridos, $\mathrm{FLA}_{2}$-Lp (443 frente a $374 \mu \mathrm{g} / \mathrm{L})$ y PCR-as $(3,85$ frente a $3,08 \mathrm{mg} / \mathrm{L})$ fueron superiores entre los sujetos que habían sufrido un ictus isquémico. Los niveles de colesterol-HDL fueron significativamente inferiores en este grupo y no se observaron diferencias en los niveles de colesterol-LDL. En el análisis realizado entre los sujetos proce- 
dentes de la muestra aleatoria los niveles de $\mathrm{FLA}_{2}$-Lp, se correlacionaron positivamente con los de colesterol-LDL, de forma negativa con los de colesterol-HDL, y no se observó ninguna correlación entre estos niveles y los de PCR-as, hallazgos similares a los publicados en otros estudios. El análisis univariado ajustado por edad, sexo y raza mostró que los niveles de $\mathrm{FLA}_{2}-\mathrm{Lp}$ en el tercil superior $(\geq 422 \mu \mathrm{g} / \mathrm{L})$ se asociaban a una mayor RR de ictus isquémico $(\mathrm{RR}=2,23$; IC $95 \%=1,48-3,34)$. Esta asociación se mantuvo después de realizar el análisis multivariado, que incluyó los factores de riesgo tradicionales y la PCR-as, con una RR de 1,97 (IC 95\% = 1,16-3,33). Al analizar la interacción entre los niveles elevados de FLA 2 -Lp y de PCR-as, se observó que la RR era de 11,38 (IC 95\% = 3,13-41,41), mientras que el riesgo era reducido en los sujetos con niveles de ambos parámetros en el rango inferior.

Los autores concluyeron que, mientras que los niveles de triglicéridos, colesterol total y sus fracciones no mostraban una asociación significativa con el riesgo de ictus isquémico, los niveles de $\mathrm{FLA}_{2}$-Lp y PCR sí se asociaban a un mayor riesgo de sufrirlo. Sugirieron que la reducción del riesgo de ictus observada en los individuos tratados con estatinas en estudios como el Heart Protection Study podía relacionarse con la disminución de la actividad de $\mathrm{FLA}_{2}$-Lp y no con la reducción de los niveles de colesterol-LDL, por lo que esta enzima se convertiría en un objetivo terapéutico independiente de los niveles lipídicos.

Este mismo grupo ha descrito que el uso combinado de los niveles de $\mathrm{FLA}_{2}$-Lp y PCR-as, junto con los factores de riesgo tradicionales, permite estimar de forma más precisa el riesgo de sufrir un ictus, al reclasificar a una parte considerable de pacientes inicialmente de bajo y medio riesgo, como de riesgo medio y alto [26]. Los investigadores demostraron que la $\mathrm{FLA}_{2}$-Lp, entre otros nuevos marcadores, permitía determinar con mayor precisión que los clásicos factores de riesgo vascular el riesgo de eventos coronarios durante un período de cinco años [27].

Un estudio realizado en 467 pacientes procedentes del Northern Manhattan Stroke Study con diagnóstico de primer ictus isquémico describió hallazgos similares. Los autores demostraron que los pacientes con niveles de $\mathrm{FLA}_{2}$-Lp en el cuartil superior, comparados con los del cuartil inferior, presentaban una RR de 2,08 (IC 05\% = 1,04-4,18) para un ictus recurrente durante el seguimiento y de 1,86 (IC $95 \%=1,01-3,42$ ) para el criterio de valoración combinado de ictus/IAM/muerte de origen vascular. Esta asociación se mantuvo al realizar el ajuste por los factores de riesgo tradicionales y por los niveles de PCR-as. Los niveles de $\mathrm{FLA}_{2}$-Lp no se asociaron a la gravedad del ictus, mientras que los de PCR-as sí se asociaron a la gravedad, así como al riesgo de muerte. Como ya se había descrito en pacientes con patología coronaria, los niveles de $\mathrm{FLA}_{2}$-Lp se correlacionaron fuertemente con los de colesterol-LDL $(r=0,27 ; p<0,001) \mathrm{y} d \mathrm{de}$ forma débil con los de PCR-as ( $r=0,09 ; p=0,045)$ [28].

En el estudio Rotterdam se evaluaron prospectivamente la incidencia y los factores de riesgo relacionados con patología discapacitante en una cohorte de base poblacional integrada por 7.983 sujetos de ambos sexos de edad superior o igual a 55 años del área de Rotterdam [13]. El diseño incluyó la realización de una entrevista basal y dos posteriores, entre los años 1990 y 1993, extrayendo subcohortes de la muestra principal para valorar determinados factores de exposición. En uno de los estudios realizados a partir de la cohorte Rotterdam, los autores determinaron la actividad basal de FLA 2 -Lp y los niveles de PCR, colesterol total y colesterol no HDL (básicamente colesterol-LDL), y su relación con el riesgo de cardiopatía coronaria e ictus isquémico en 1.820 participantes. Se siguió a los sujetos durante una media de 7,2 años. Después del análisis ajustado por sexo, edad y factores de riesgo cardiovascular tradicionales, e independientemente de los niveles de colesterol-HDL y no HDL, los niveles elevados de $\mathrm{FLA}_{2}$-Lp se asociaron a un mayor riesgo relativo, tanto de cardiopatía coronaria $(\mathrm{RR}=1,39,1,99$ y 1,97 al comparar el primer cuartil con el segundo, tercero, y cuarto, respectivamente) como de ictus isquémico ( $R R=1,08,1,58$ y 1,97 al comparar el primer cuartil con el segundo, tercero, y cuarto, respectivamente). Como en otros estudios, la actividad de la FLA 2 -Lp fue independiente del sexo y de los niveles de PCR.

Los autores concluyeron que la actividad de la $\mathrm{FLA}_{2}$-Lp es un factor predictivo de cardiopatía coronaria y de ictus isquémico en la población general, independientemente de los factores de riesgo cardiovascular clásicos y de la PCR. De forma interesante, la asociación se mantuvo en todo el rango de niveles de colesterol total y no HDL.

Empleando la mencionada subcohorte del estudio Rotterdam, se valoró la relación entre la $\mathrm{FLA}_{2}$-Lp y el grado de ateroesclerosis subclínica mediante el examen ultrasonográfico de ambas arterias carótidas, la razón de presión arterial sistólica entre la extremidad superior y el tobillo, y la presencia de calcificación aórtica en la radiografía lateral de tórax. Los autores describieron una asociación entre la actividad de la FLA 2 -Lp y el sexo masculino, el índice de masa corporal, la presión arterial sistólica y el colesterol total, y una relación inversa con los niveles de colesterol-HDL. Al comparar los valores de FLA 2 -Lp situados en el tercil superior con los del tercil inferior, los primeros se asociaron de forma significativa a la presencia de ateroesclerosis o a la suma de ateroesclerosis carotídea y aortoilíaca. La asociación se mantuvo después de realizar el ajuste por edad y sexo - odds ratio $(\mathrm{OR})=1,97$ en ambos casos-, pero perdió su significación al realizarlo por los niveles de colesterol total y HDL. Los autores concluyen que, dada la discordancia entre la relación demostrada entre FLA $_{2}$-Lp y los eventos cardiovasculares, y la ausencia de asociación entre FLA $_{2}$-Lp y la ateroesclerosis extracoronaria subclínica, el papel de la enzima en fases preclínicas no está definido [29].

Se han realizado estudios sobre el riesgo de ictus en grupos específicos, como las mujeres posmenopáusicas sometidas o no a tratamiento hormonal sustitutivo. Tras evaluar a 929 mujeres con ictus y a 935 controles, y de realizar un ajuste por los niveles de PCR y los factores de riesgo tradicionales, WassertheilSmoller et al describieron una asociación entre $\mathrm{FLA}_{2}$-Lp y la incidencia de ictus. La OR global fue de 1,08 (IC 95\% = 0,75$1,55)$ para las mujeres con niveles en el cuartil superior respecto a las mujeres con niveles en el inferior. En el subgrupo que no recibía terapia hormonal, la OR fue de 1,55 (IC 95\% = 1,05-2,28) y aumentaba a 2,26 (IC 95\% = 1,55-3,35) si la PCR estaba elevada, mientras que en el subgrupo tratado se perdía la asociación entre $\mathrm{FLA}_{2}$-Lp e ictus $(\mathrm{OR}=0,7$; IC 95\% = 0,42-1,17) [30].

Estos resultados, sin embargo, no han sido confirmados por otros autores. En un interesante trabajo que incluyó a 28.263 mujeres sanas de mediana edad, no se detectó asociación entre la $\mathrm{FLA}_{2}$-Lp y el riesgo de eventos cardiovasculares (patología coronaria o ictus) durante los tres años de seguimiento. El riesgo sí se relacionó con los niveles de PCR, y los autores concluyeron que la $\mathrm{FLA}_{2}$-Lp no es un factor de predicción del riesgo cardiovascular en mujeres no seleccionadas [31].

En la tabla II se resumen los principales estudios publicados. 
Tabla II. Estudios en los que se evalúa la relación entre $\mathrm{FLA}_{2}$-Lp y el riesgo de ictus isquémico y otras patologías vasculares.

\begin{tabular}{|c|c|c|c|c|c|c|}
\hline Ref. & Diseño del estudio & ECV valorados & $\begin{array}{l}\text { Características } \\
\text { de los individuos }\end{array}$ & $\begin{array}{l}\mathrm{N} .^{\circ} \text { de } \\
\text { individuos }\end{array}$ & $\begin{array}{l}\text { Seguimiento } \\
\text { medio (años) }\end{array}$ & Riesgo \\
\hline [31] & Casos y controles & $\begin{array}{l}\text { Mortalidad, } \\
\text { IAM no fatal, ictus }\end{array}$ & Mujeres sanas & 28.263 & 3 & $\begin{array}{l}\text { Ausencia de asociación entre } F L A_{2}-L p \text { y mortalidad, } \\
\text { riesgo de IAM no fatal e ictus isquémico }\end{array}$ \\
\hline [14] & Cohorte prospectiva & $\begin{array}{l}\text { Incidencia de } \\
\text { ictus isquémico }\end{array}$ & Individuos sanos & 12.762 & 6 & $\mathrm{RR}=1,97(\mathrm{IC} 95 \%=1,16-3,33)$ \\
\hline [13] & Cohorte prospectiva & $\begin{array}{l}\text { Incidencia de ictus } \\
\text { y eventos coronarios }\end{array}$ & Población general & 1.820 & 7.2 & $\begin{array}{l}\mathrm{RR} \text { de } \mathrm{Cl}=1,39,1,99, \text { y } 1,97 ; \mathrm{RR} \text { de ictus }=1,08, \\
1,58 \text { y } 1,97 \text { al comparar } \mathrm{Q} 1 \text { con } \mathrm{Q} 2, \mathrm{Q} 3 \text { y } \mathrm{Q} 4, \\
\text { respectivamente }\end{array}$ \\
\hline [16] & Cohorte prospectiva & $\begin{array}{l}\text { Angina inestable, } \\
\text { IAM e ictus }\end{array}$ & Pacientes con $\mathrm{Cl}$ & 504 & 4 & $\begin{array}{l}\mathrm{RR}=1,28(\mathrm{IC} 95 \%=1,06-1,54) \text { por cada } \mathrm{DE} ; \\
\text { el riesgo se refiere al total de eventos, no se } \\
\text { diferencian ictus de } \mathrm{Cl}\end{array}$ \\
\hline [28] & Cohorte prospectiva & $\begin{array}{l}\text { Recurrencia de } \\
\text { ictus y mortalidad }\end{array}$ & $\begin{array}{l}\text { Primer ictus } \\
\text { isquémico }\end{array}$ & 467 & 4 & $\begin{array}{l}\text { RR de ictus recurrente: } 2,08 \text { (IC } 95 \%=1,04-4,18) \\
\text { RR de ictus/IAM/muerte vascular: } 1,86 \text { (IC } 95 \%= \\
1,01-3,42)\end{array}$ \\
\hline [32] & Casos y controles & $\begin{array}{l}\text { Muerte, eventos } \\
\text { coronarios e ictus }\end{array}$ & Pacientes con $\mathrm{Cl}$ & 1.206 & 4,5 & $\begin{array}{l}\text { RR de ECV }=1,81(\mathrm{IC} 95 \%=0,94-3,49) \\
\text { en el T superior de } \mathrm{FLA}_{2} \text { - } L \mathrm{p}\end{array}$ \\
\hline [37] & Cohorte prospectiva & $\begin{array}{l}\text { Muerte, eventos } \\
\text { coronarios e ictus }\end{array}$ & $\begin{array}{l}\text { Pacientes con } \mathrm{Cl} \text { tra- } \\
\text { tados con estatinas }\end{array}$ & 3.648 & 2 & $\begin{array}{l}\text { RR de ECV al comparar el quintil superior } \\
\text { y el inferior }=1,33(\text { IC } 95 \%=1,01-1,74)\end{array}$ \\
\hline [39] & Cohorte prospectiva & $\begin{array}{l}\text { Muerte, eventos } \\
\text { coronarios e ictus }\end{array}$ & Población general & 765 & 10 & $\begin{array}{l}\text { RR de ECV }=1,4 \text { (IC } 95 \%=0,8-2,6) \text { y } 2,6 \\
\text { (IC } 95 \%=1,4-4,6) \text { al comparar T3 con T2 y } 1\end{array}$ \\
\hline [40] & Cohorte prospectiva & $\begin{array}{l}\text { Muerte, eventos } \\
\text { coronarios e ictus }\end{array}$ & Población general & 4.480 & 10 & $\begin{array}{l}\text { RR de ECV = 1,54 (IC 95\% = 1,07-2,24) } \\
\text { al comparar T superior e inferior; RR = } 1,97 \\
\text { (IC } 95 \%=1,34-2,9) \text { si existe síndrome metabólico }\end{array}$ \\
\hline [22] & Cohorte prospectiva & $\begin{array}{l}\text { Muerte, eventos } \\
\text { coronarios e ictus }\end{array}$ & $\begin{array}{l}\text { Pacientes con } \\
\text { Cl estable }\end{array}$ & 3.766 & 4,8 & $\begin{array}{l}\text { RR de } E C V=1,41(I C \text { C 95\% = 1,17-1,7) } \\
\text { al comparar Q1 con Q4 }\end{array}$ \\
\hline [30] & Casos y controles & $\begin{array}{l}\text { Incidencia de } \\
\text { ictus isquémico }\end{array}$ & $\begin{array}{l}\text { Mujeres posmeno- } \\
\text { páusicas con ictus }\end{array}$ & $\begin{array}{l}929 \text { casos } \\
935 \text { control }\end{array}$ & 5,6 &  \\
\hline
\end{tabular}

$\mathrm{Cl}$ : cardiopatía isquémica; DE: desviación estándar; ECV: eventos cardiovasculares; FLA 2 -Lp: fosfolipasa $\mathrm{A}_{2}$ asociada a lipoproteínas; IAM: infarto agudo de miocardio; IC: intervalo de confianza; OR: odds ratio; Q (Q1-Q4): cuartil (cuartiles 1-4); RR: razón de riesgos; T (T1-T3): tercil (terciles 1-3).

\section{IMPLICACIONES CLÍNICAS}

Aunque existen estudios que muestran resultados contradictorios, la mayor parte de los publicados indican que la FLA2-Lp, determinada como masa o actividad, se asocia de forma independiente al desarrollo de ateroesclerosis coronaria y extracoronaria, a la incidencia de patología coronaria (primer evento o recurrencia), a la incidencia de ictus isquémico (primer ictus o recurrente) y a la mortalidad de causa cardiovascular. Las medidas de riesgo descritas al comparar los valores inferiores con los superiores del rango son generalmente modestas e inferiores a 2 . La asociación parece ser independiente de la presencia de hipercolesterolemia, de los factores de riesgo cardiovascular clásicos y de los niveles de otros marcadores, como la PCR-as. La determinación conjunta de $\mathrm{FLA}_{2}$-Lp y PCR permite mejorar la discriminación del riesgo [26]. La coherencia entre estudios ha favorecido que la Food and Drug Administration estadounidense aprobara en 2005 el uso del test PLAC para estimar el riesgo coronario y de ictus isquémico de mecanismo ateroesclerótico en pacientes de riesgo.

Una de las limitaciones que existen en las diferentes determinaciones de los niveles de $\mathrm{FLA}_{2}$-Lp es la falta de uniformidad entre los estudios, ya que los riesgos descritos se han establecido para comparaciones entre terciles superiores e inferiores, cuartiles superiores e inferiores, o incrementos en la desviación estándar. Por este motivo, investigadores de la empresa que comercializa el enzimoinmunoensayo PLAC han recomendado establecer el percentil $50(235 \mathrm{ng} / \mathrm{mL})$ como punto de corte a partir del cual considerar que el paciente presenta un mayor riesgo cardiovascular, independientemente de los factores de riesgo establecidos, y de los niveles de colesterol-LDL y de PCR-as [43].

La enzima $\mathrm{FLA}_{2}$-Lp parece participar en la desestabilización de la placa de ateroma, al favorecer los procesos inflamatorios locales, necesarios para su posterior crecimiento y rotura. Estos datos experimentales y la evidencia procedente de estudios epidemiológicos han justificado el desarrollo clínico de dos fármacos inhibidores de la enzima [44]. Un ensayo clínico de fase II que comparó darapladib (SB-480848) con placebo mostró una reducción dependiente de la dosis de la actividad plasmática de $\mathrm{FLA}_{2}$-Lp y de otros marcadores inflamatorios, como la interleucina 6 y la PCR, en pacientes con cardiopatía isquémica tratados con atorvastatina, sin que se observaran efectos adversos destacables [45]. El efecto de este fármaco sobre la actividad plasmática de la $\mathrm{FLA}_{2}$-Lp se evaluará próximamente en un ensayo clínico de fase II realizado en pacientes dislipémicos, cuya etapa de reclutamiento no ha comenzado.

Otro de los fármacos en estudio es varespladib (A-001). Actualmente, el ensayo clínico FRANCIS (Fewer Recurrent Acute coronary events with Near-term Cardiovascular Inflammation Suppression) recluta pacientes con el objetivo de examinar el efecto de su administración durante las primeras 96 horas después del inicio de un síndrome coronario agudo [46]. El tratamiento activo o el placebo se asocian a atorvastatina durante seis meses y, posteriormente, se valoran los niveles de marcado- 
res de inflamación y un objetivo formado por la combinación de la incidencia de eventos cardiovasculares (IAM no fatal, angina inestable, necesidad de procedimiento de revascularización coronaria, muerte de causa cardiovascular e ictus no fatal). La inclusión del ictus entre los criterios de valoración hace que los resultados de este estudio, que se esperan para el segundo trimestre de 2009, sean de especial interés para los neurólogos.

Otro ensayo clínico de fase II, el SPIDER-PCI ( $s P L A_{2}$ Inhibition to Decrease Enzyme Release After PCI), está reclutando pacientes para evaluar varespladib en la prevención de la lesión miocárdica posterior a la angioplastia coronaria. Recientemente, se ha suspendido el ensayo clínico de fase II IMPACTS (Investigation of the Modulation of Phospholipase in Acute Chest Syndrome), que reclutaba pacientes diagnosticados de anemia de células falciformes que presentaban crisis vasooclusivas. Se han concluido dos ensayos clínicos de fase II, el PLASMA (Phospholipase Levels And Serological Markers of Atherosclerosis) y el PLASMA-2, que evaluaron la reducción de los niveles de marcadores de inflamación en pacientes con patología coronaria tratados con diferentes regímenes de varespladib.

Desde un punto de vista práctico, deberá definirse el papel preciso de los inhibidores de $\mathrm{FLA}_{2}$-Lp, ya que fármacos hipolipemiantes actualmente disponibles, como atorvastatina o fenofibrato, pueden reducir la actividad plasmática de la $\mathrm{FLA}_{2}-\mathrm{Lp}$ entre un $20-30 \%$ sin actuar sobre la síntesis de la enzima, y se han publicado estudios que demuestran la infrautilización de las estatinas en pacientes de riesgo [47]. Posiblemente, este efecto se debe a la reducción de magnitud equivalente que ejercen so- bre los niveles de colesterol-LDL [48-50]. Sin embargo, determinados estudios previos, que relacionan el efecto clínico de las estatinas con la reducción de los niveles de $\mathrm{FLA}_{2}$-Lp y no con la del colesterol-LDL, sugieren que la interrelación entre estos fármacos, la $\mathrm{FLA}_{2}$-Lp y la disminución del riesgo vascular es compleja y no está definitivamente aclarada [14].

En resumen, la $\mathrm{FLA}_{2}$-Lp puede determinarse como un complemento de los restantes factores reconocidos de riesgo vascular. Existe una serie de cuestiones sin clarificar, como su papel definitivo en la fisiopatología de la ateroesclerosis, el papel de los diferentes polimorfismos genéticos existentes, los valores de corte en los diferentes grupos de riesgo, las indicaciones para su determinación y las opciones de manipulación terapéutica de la enzima. El volumen de estudios en marcha es importante, especialmente en el campo de la cardiopatía isquémica. Aunque parte de estos resultados podrán aplicarse a la patología vascular cerebral isquémica, este campo afronta retos específicos. Por ejemplo, desconocemos la asociación de la FLA 2 -Lp con los diferentes subtipos de ictus, así como su posible relación con la recurrencia, mortalidad y morbilidad posteriores. Si se consideran los ictus de mecanismo aterotrombótico, no se tienen datos acerca de la evolución de la ateromatosis carotídea y el riesgo de ictus en función del nivel de $\mathrm{FLA}_{2}$-Lp, así como de su papel en la ateromatosis intracraneal. Finalmente, es posible que el uso de inhibidores específicos de la enzima, junto con la terapia hipolipemiante clásica, sea uno de los próximos objetivos de la investigación clínica en la prevención del ictus isquémico.

\section{BIBLIOGRAFÍA}

1. Fernández-Gómez FJ, Hernández F, Argandoña L, Galindo MF, Segura T, Jordán J. Farmacología de la neuroprotección en el ictus isquémico agudo. Rev Neurol 2008; 47: 253-60.

2. Garza CA, Montori VM, McConnell JP, Somers VK, Kullo IJ, LópezJiménez F. Association between lipoprotein-associated phospholipase $\mathrm{A}_{2}$ and cardiovascular disease: a systematic review. Mayo Clin Proc 2007; 82: 159-65.

3. Sudhir K. Clinical review: lipoprotein-associated phospholipase $\mathrm{A}_{2}$, a novel inflammatory biomarker and independent risk predictor for cardiovascular disease. J Clin Endocrinol Metab 2005; 90: 3100-5.

4. Zalewski A, Macphee C. Role of lipoprotein-associated phospholipase $\mathrm{A}_{2}$ in atherosclerosis. Biology, epidemiology, and possible therapeutic target. Arterioscler Thromb Vasc Biol 2005; 25: 923-31.

5. Jenny NS. Lipoprotein-associated phospholipase $\mathrm{A}_{2}$ : novel biomarker and causal mediator of atherosclerosis? Arterioscler Thromb Vasc Biol 2006; 26: 2417-8.

6. Lindsberg PJ, Hallenbeck JM, Feuerstein G. Platelet-activating factor in stroke and brain injury. Ann Neurol 1991; 30: 117-29.

7. Feuerstein G, Yue TL, Lysko PG. Platelet-activating factor. A putative mediator in central nervous system injury? Stroke 1990; 21 (Suppl 11): S90-4.

8. Six DA, Dennis EA. The expanding superfamily of phospholipase $\mathrm{A}_{2}$ enzymes: classification and characterization. Biochim Biophys Acta 2000; 1488: 1-19.

9. Birukov KG, Bochkov VN, Birukova AA. Epoxycyclopentenone-containing oxidized phospholipids restore endothelial barrier function via Cdc42 and Rac. Cir Res 2004; 95: 892-901.

10. Bochkov VN, Leitinger N. Anti-inflammatory properties of lipid oxidation products. J Mol Med 2003; 81: 613-26.

11. Yamada Y, Izawa $H$, Ichihara S, Takatsu F, Ishihara H, Hirayama H, et al. Prediction of the risk of myocardial infarction from polymorphisms in candidate genes. N Engl J Med 2002; 347: 1916-23.

12. Abuzeid AM, Hawe E, Humphries SE, Talmud PJ, HIFMECH Study Group. Association between the Ala379Val variant of the lipoproteinassociated phospholipase $A_{2}$ and risk of myocardial infarction in the north and south of Europe. Atherosclerosis 2003; 168: 283-8.

13. Oei H, Van der Meer I, Hofman A, Koudstaal PJ, Stijnen T, Breteler $\mathrm{MMB}$, et al. Lipoprotein-associated phospholipase $\mathrm{A}_{2}$ activity is associated with risk of coronary heart disease and ischemic stroke: the Rotterdam study. Circulation 2005; 111: 570-5.
14. Ballantyne CM, Hoogeveen RC, Bang H, Coresh J, Folsom AR, Chambless LE, et al. Lipoprotein-associated phospholipase $\mathrm{A}_{2}$, high-sensitivity C-reactive protein, and risk of incident ischemic stroke in middle-aged men and women in the Atherosclerosis Risk in Communities (ARIC) study. Arch Intern Med 2005; 165: 2479-84.

15. Packard CJ, O'Reilly D, Caslake M, McMahon AD, Ford I, Cooney J, et al. Lipoprotein-associated phospholipase $\mathrm{A}_{2}$ as an independent predictor of coronary heart disease. N Engl J Med 2000; 343: 1148-55.

16. Brilakis ES, McConnell JP, Lennon RJ, Elesber AA, Meyer JG, Berger PB. Association of lipoprotein-associated phospholipase $\mathrm{A}_{2}$ levels with coronary artery disease risk factors, angiographic coronary artery disease, and major adverse events at follow-up. Eur Heart J 2005; 26: 137-44.

17. Oldgren J, James S, Siegbahn A, Wallentin L. Lipoprotein-associated phospholipase $\mathrm{A}_{2}$ does not predict mortality or new ischaemic events in acute coronary syndrome patients. Eur Heart J 2007; 28: 699-704.

18. Gerber Y, McConnell JP, Jaffe AS, Weston SA, Killian JM, Roger VL. Lipoprotein-associated phospholipase $\mathrm{A}_{2}$ and prognosis alter myocardial infarction in the community. Arterioscler Thromb Vasc Biol 2006; 26: $2517-22$

19. Khuseyinova N, Imhof A, Rothenbacher D, Trischler G, Kuelb S, Scharnagl $\mathrm{H}$, et al. Association between Lp-PLA ${ }_{2}$ and coronary artery disease: focus on its relationship with lipoproteins and markers of inflammation and hemostasis. Atherosclerosis 2005; 182: 181-8.

20. Daniels LB, Laughlin GA, Sarno MJ, Bettencourt R, Wolfert RL, Barrett-Connor E. Lipoprotein-associated phospholipase $\mathrm{A}_{2}$ is an independent predictor of incident coronary heart disease in an apparently healthy older population: the Rancho Bernardo Study. J Am Coll Cardiol 2008; 51: 913-9.

21. Möckel M, Müller R, Vollert JO, Müller C, Danne O, Gareis R, et al. Lipoprotein-associated phospholipase $\mathrm{A}_{2}$ for early risk stratification in patients with suspected acute coronary syndrome: a multi-marker approach: the North Wuerttemberg and Berlin Infarction Study-II (NOBIS-II). Clin Res Cardiol. 2007; 96: 604-12.

22. Sabatine MS, Morrow DA, O'Donoghue M, Jablonksi KA, Rice MM, Solomon S, et al. Prognostic utility of lipoprotein-associated phospholipase $\mathrm{A}_{2}$ for cardiovascular outcomes in patients with stable coronary artery disease. Arterioscler Thromb Vasc Biol 2007; 27: 2463-9.

23. Raichlin E, McConnell J, Bae JH, Kremers WK, Lerman A, Frantz R. Lipoprotein-associated phospholipase $\mathrm{A}_{2}$ predicts progression of car- 
diac allograft vasculopathy and increased risk of cardiovascular events in heart transplant patients. Transplantation 2008; 85: 963-8.

24. Satoh K, Imaizumi T, Kawamura Y, Yoshida H, Takamatsu S, Mizono $\mathrm{S}$, et al. Activity of platelet-activating factor (PAF) acetylhydrolase in plasma from patients with ischemic cerebrovascular disease. Prostaglandins 1988; 35: 685-98.

25. Satoh K, Yoshida H, Imaizumi T, Takamatsu S, Mizuno S. Platelet-activating factor acetylhydrolase in plasma lipoproteins from patients with ischemic stroke. Stroke 1992; 23: 1090-2.

26. Nambi V, Hoogeven RC, Chambles L, Hu Y, Bang H, Coresh J, et al. Lipoprotein-associated phospholipase $\mathrm{A}_{2}$ and high-sensitivity C-reactive protein improve the stratification of ischemic stroke risk in the Atherosclerosis Risk in Communities (ARIC) study. Stroke 2009; 40: 376-81.

27. Folsom AR, Chambless LE, Ballantyne CM, Coresh J, Heiss G, Wu $\mathrm{KK}$, et al. An assessment of incremental coronary risk prediction using C-reactive protein and other novel risk markers. Arch Intern Med 2006; 166: $1368-73$.

28. Elkind MS, Tai W, Coates K, Paik MC, Sacco RL. High-sensitivity Creactive protein, lipoprotein-associated phospholipase $\mathrm{A}_{2}$, and outcome alter ischemic stroke. Arch Intern Med 2006; 166: 2073-80.

29. Kardys I, Oei H, Van der Meer I, Hofman A, Breteler MMB, Witteman JCM. Lipoprotein-associated phospholipase $\mathrm{A}_{2}$ and measures of extracoronary atherosclerosis: the Rotterdam study. Arterioscler Thromb Vasc Biol 2006; 26: 631-6.

30. Wassertheil-Smoller S, Kooperberg C, McGinn AP, Kaplan RC, Hsia J, Hendrix SL, et al. Lipoprotein-associated phospholipase $\mathrm{A}_{2}$, hormone use, and the risk of ischemic stroke in postmenopausal women. Hypertension 2008; 51: 1115-22.

31. Blake GJ, Dada N, Fox JC, Manson JE, Ridker PM. A prospective evaluation of lipoprotein-associated phospholipase $\mathrm{A}_{2}$ levels and the risk of future cardiovascular events in women. J Am Coll Cardiol 2001; 38: 1302-6.

32. Koenig W, Twardella D, Brenner H, Rothenbacher D. Lipoprotein-associated phospholipase $\mathrm{A}_{2}$ predicts future cardiovascular events in patients with coronary heart disease independently of traditional risk factors, markers o inflammation, renal function, and hemodynamic stress. Arterioscler Thromb Vasc Biol 2006; 26: 1586-93.

33. Winkler K, Winkelmann BR, Scharnagl H, Hoffmann MM, Grawitz AB, Nauck M, et al. Platelet-activating Factor acetylhydrolase activity indicates angiographic coronary artery disease independently of systemic inflammation and other risk factors: the Ludwigshafen Risk and Cardiovascular Health Study. Circulation 2005; 111; 980-7.

34. May HT, Horne BD, Anderson JL, Wolfert RL, Muhlestein JB, Renlund DG, et al. Lipoprotein-associated phospholipase $\mathrm{A}_{2}$ independently predicts the angiographic diagnosis of coronary artery disease and coronary death. Am Heart J 2006; 152: 997-1003.

35. Koenig W, Khuseyinova N, Löwel H, Trischler G, Meisinger C. Lipoprotein-associated phospholipase $\mathrm{A}_{2}$ adds to risk prediction of incident coronary events by C-reactive protein in apparently healthy middle-aged men from the general population. Results from the 14year follow-up of a large cohort from Southern Germany. Circulation 2004; 110: 1903-8.

36. Corsetti JP, Rainwater DL, Moss AJ, Zareba W, Sparks CE. High lipoprotein-associated phospholipase $\mathrm{A}_{2}$ is a risk factor for recurrent coronary events in postinfarction patients. Clin Chem 2006; 52: 1331-8.
37. O'Donoghue M, Morrow DA, Sabatine MS, Murphy SA, McCabe CH, Cannon CP, et al. Lipoprotein-associated phospholipase $\mathrm{A}_{2}$ and its association with cardiovascular outcomes in patients with acute coronary syndromes in the PROVE IT-TIMI 22 (Pravastatin or Atorvastatin Evaluation and Infection Therapy-Thrombolysis In Myocardial Infarction) Trial. Circulation 2006; 113: 1745-52.

38. Iribarren C, Gross MD, Darbinian JA, Jacobs DR, Sidney S, Loria CM. Association of lipoprotein-associated phospholipase $\mathrm{A}_{2}$ mass and activity with calcified coronary plaque in young adults. The CARDIA Study. Arterioscler Thromb Vasc Biol 2005; 25: 216-21.

39. Kiechl S, Willeit J, Mayr M, Viehweider B, Oberhollenzer M, Kronenberg F, et al. Oxidized phospholipids, lipoprotein(a), lipoprotein-associated phospholipase $\mathrm{A}_{2}$ activity, and 10-year cardiovascular outcomes. Prospective results from the Bruneck Study. Arterioscler Thromb Vasc Biol 2007; 27: 1788-95

40. Persson M, Hedblad B, Nelson JJ, Berglund G. Elevated Lp-PLA, levels add prognostic information to the metabolic syndrome on incidence of cardiovascular events among middle-aged nondiabetic subjects. Arterioscler Thromb Vasc Biol 2007; 27: 1411-6.

41. Winkler K, Hoffmann MM, Winkelmann BR, Friedrich I, Schafer G, Seelhorst $\mathrm{U}$, et al. Lipoprotein-associated phospholipase $\mathrm{A}_{2}$ predicts 5 year cardiac mortality independently of established risk factors and adds prognostic information in patients with low and medium highsensitivity C-reactive protein (The Ludwigshafen Risk and Cardiovascular Health Study). Clin Chem 2007; 53: 1440-7.

42. Blankenberg S, Stengel D, Rupprecht HJ, Bickel C, Meyer J, Cambien $\mathrm{F}$, et al. Plasma PAF-acetylhydrolase in patients with coronary artery disease: results of a cross-sectional analysis. J Lipid Res 2003; 44: 1381-6.

43. Lanman RB, Wolfert RL, Fleming JK, Jaffe AS, Roberts WL, Warnick GR, et al. Lipoprotein-associated phospholipase $\mathrm{A}_{2}$ : review and recommendation of a clinical cut point for adults. Prev Cardiol 2006; 9: 138-43.

44. Clinical Trials. URL: http://www.clinicaltrials.gov/ct2/results?term= phospholipase. [29.01.2009].

45. Mohler ER III, Ballantyne CM, Davidson MH. The effect of darapladib on plasma lipoprotein-associated phospholipase $\mathrm{A}_{2}$ activity and cardiovascular biomarkers in patients with stable coronary heart disease or coronary heart disease risk equivalent: the results of a multicenter, randomized, double-blind, placebo-controlled study. J Am Coll Cardiol 2008; 51: 1632-41.

46. Anthera Pharmaceuticals. URL: http://www.anthera.com/francis.html. [29.01.2009].

47. Carneado-Ruiz J, Sánchez-Payá J, Alfaro-Sáez A, Lezcano-Rodas M, Berenguer-Ruiz L, Mira-Berenguer F, et al. Antecedente del uso de estatinas en el ictus: oportunidad de mejora en el paciente con alto riesgo vascular por aterotrombosis. Rev Neurol 2007; 45: 449-55.

48. Tsimihodimos V, Karabina SA, Tambaki AP, Bairaktari E, Goudevenos JA, Chapman MJ, et al. Atorvastatin preferentially reduces LDL-associated platelet-activating factor acetylhydrolase activity in dyslipemias of type IIA and type IIB. Arterioscler Thromb Vasc Biol 2002; 22: 306-11.

49. Castilla-Guerra L, Fernández-Moreno MC, López-Chozas JM, Jiménez-Hernández MD. Estatinas y enfermedad cerebrovascular: nuevas perspectivas en la prevención del ictus. Rev Neurol 2007; 44: 95-100.

50. Tsimihodimos V, Kakafica A, Tambaki AP, Bairaktari E, Chapman MJ, Elisaf M, et al. Fenofibrate induces HDL-associated PAF-AH but attenuates enzyme activity associated with apoB-containing lipoproteins. J Lipid Res 2003; 44: 927-34.

\section{LIPOPROTEIN-ASSOCIATED PHOSPHOLIPASE $A_{2}$ AND STROKE}

Summary. Introduction. Lipoprotein-associated phospholipase $A_{2}\left(L p-P L A_{2}\right)$ is an enzyme member of $A_{2}$ phosholipases superfamily. The $80 \%$ circulates bound to LDL-cholesterol and levels of both parameters are positively correlated. The main function of this enzyme is to hydrolyze oxidized phospholipids contained in LDL-cholesterol forming pro-inflammatory products in atheroma plaque. Several studies related raised $\mathrm{Lp}-\mathrm{PLA} \mathrm{A}_{2}$ and vascular risk. Aim. To review available data relating Lp-PLA $A_{2}$ and stroke. Development. Different cohorts and cases and controls studies showed an association between higher levels of $\mathrm{Lp}_{-} \mathrm{PLA}_{2}$ and the risk of suffering: first cardiovascular event (coronary heart disease, ischemic stroke) or its recurrence; progression of coronary damage evaluated using cardiac computed tomography; or death of cardiovascular cause. Published risk measurements are less than 2 and it has been demonstrated that raised levels of enzyme are risk factor independent of classic cardiovascular risk factors and non-HDL cholesterol and C-reactive protein levels. The clinical data and the knowledge of the role of Lp-PLA 2 in plaque destabilization justified the development of inhibitor drugs. Conclusions. According to current evidence, patients in the higher range of Lp-PLA $A_{2}$ may be considerate at higher risk of suffering a vascular event. The application to stroke needs a better definition of the pathophysiologic role and the knowledge of phase II clinical trials currently under development in coronary heart disease. [REV NEUROL 2009; 49: 88-94]

Key words. $A_{2}$ phospholipase. Atherosclerosis. Cholesterol. Coronary heart disease. C reactive protein. Stroke. 